Article

\title{
Resveratrol-Loaded Solid Lipid Nanoparticle Supplementation Ameliorates Physical Fatigue by Improving Mitochondrial Quality Control
}

\author{
Jingyu Sun ${ }^{1, \dagger}$, Yunhe Zhou ${ }^{1,+}\left(\mathbb{D}\right.$, Yajuan $\mathrm{Su}^{2}$, Sheng $\mathrm{Li}^{2}$, Jingmei Dong ${ }^{1}{ }^{\mathbb{D}}$, Qing $\mathrm{He}^{2}$, \\ Yang Cao ${ }^{1}$, Tianfeng Lu ${ }^{1, *}$ and Lili Qin ${ }^{1, *}$ \\ 1 Sports and Health Research Center, Department of Physical Education, Tongji University, Shanghai 200092, \\ China; jysun@tongji.edu.cn (J.S.); maggie211@tongji.edu.cn (Y.Z.); djm1969@tongji.edu.cn (J.D.); \\ caoyang@tongji.edu.cn (Y.C.) \\ 2 School of Life Sciences and Technology, Tongji University, Shanghai 200092, China; \\ 18338691923@163.com (Y.S.); lisheng1209@163.com (S.L.); 1831521@tongji.edu.cn (Q.H.) \\ * Correspondence: sytyltf@126.com (T.L.); qinlili@tongji.edu.cn (L.Q.); \\ Tel.: +86-21-6598-5242 (T.L.); +86-21-6598-1711 (L.Q.) \\ + These authors contributed equally to this article.
}

Received: 2 September 2019; Accepted: 21 October 2019; Published: 25 October 2019

\begin{abstract}
Resveratrol (RSV) has various pharmacological effects; however, few studies have directly addressed the possible antifatigue effects of long-term endurance exercise. The clinical use of RSV is limited by its poor water solubility and extremely short plasma half-life. Solid lipid nanoparticles (SLNs) are considered as reasonable drug delivery systems to overcome some of these drawbacks and expand its applications. In this study, RSV-SLNs were successfully prepared through emulsification and low-temperature solidification. Results showed that RSV-SLN supplementation effectively enhanced endurance performance. RSV-SLN supplementation might enhance mitochondrial function by ameliorating mitochondrial quality control (QC), which was superior to RSV application. These results revealed an unexpected role of RSV-SLN compared with RSV in terms of linking nutrient deprivation to mitochondrial oxidant production through mitochondrial QC. A mitochondrion-mediated pathway was likely involved in RSV-SLN, thereby improving endurance performance. Overall, this study highlighted new possibilities for anti-physical-fatigue strategies.
\end{abstract}

Keywords: resveratrol; solid lipid nanoparticles; endurance exercise; mitochondrial nutrients; mitochondrial quality control

\section{Introduction}

Physical fatigue and mental fatigue are two main aspects of fatigue. Physical fatigue is often accompanied by the deterioration of physical function [1]. Exhaustive exercise-induced mitochondrial dysfunction may result in physical fatigue [2]. Mitochondrial nutrients protect organelles from chronic and repeated exercise or excessive fatigue-induced damage and maintain metabolic homeostasis. Therefore, scientists are actively exploring natural products to reduce oxidative damage caused by exercise and fight against physical fatigue [3]. Resveratrol (3-5-4'-trihydroxy-trans-stilbene, RSV), a polyphenol compound, has various pharmacological effects, including improvement of mitochondrial function, prevention of obesity and obesity-related diseases [4], suppression of inflammation [5], and protection against oxidative stress [6]. However, to our knowledge, few studies have directly addressed the possible anti-physical-fatigue effects of RSV. The pharmacokinetic properties of RSV are less favorable because of the poor water solubility of RSV. Beyond that, RSV metabolism is rapid and extensive [7], and its plasma half-life is short [8]. A reasonable strategy is needed to propose and 
expand the applications of RSV to resolve some of these problems. Solid lipid nanoparticles (SLNs) are considered as an efficient drug delivery system because of their good physicochemical properties $[9,10]$. SLNs, which can be metabolized by many organisms, can modulate drug release [11-13]. SLNs can efficiently protect encapsulated resveratrol drugs in the biological environment and improve their physiochemical properties [14-16]. Hence, this study evaluated the effect of RSV-loaded SLN (RSV-SLN) supplementation on exercise performance to explore its possible mechanisms.

Maintaining mitochondrial function involves mitochondrial biogenesis, mitophagy, fusion, and fission. The integration of this series of processes reflects mitochondrial quality control (QC). We hypothesized that chronic fatigue states induced by excessive endurance exercise might be linked to injured mitochondrial QC. This study was the first to explore the effects of RSV-loaded SLNs on gene regulation that involves mitochondrial QC following excessive endurance exercise in mice. These data improved the understanding of the role of RSV in a nanometer form as mitochondrial nutrition during successive sessions of prolonged endurance exercise.

\section{Materials and Methods}

\subsection{Preparation of RSV-SLNS}

RSV-SLNs were produced by emulsification and low-temperature solidification. In brief, RSV (150 mg) (Aladdin Industrial Corporation, Shanghai, China), lecithin (100 mg), and stearic acid (200 mg) (Shanghai Chemical Reagent Company, Shanghai, China) were dissolved in $10 \mathrm{~mL}$ of chloroform in glass bottles as an organic phase through ultrasound. Myrj 52 (Sigma-Aldrich Co., St Louis, MO, USA) was dissolved in $30 \mathrm{~mL}$ of distilled water and heated to $75 \pm 2{ }^{\circ} \mathrm{C}$ in a water bath as an aqueous phase. Under $1000 \mathrm{rpm}$ mechanical stirring, the organic phase was injected into the hot water phase, and the solution was kept at $75{ }^{\circ} \mathrm{C}$ at the same stirring speed to remove organic solvents. Approximately $5 \mathrm{~mL}$ of condensed solvent remained after the organic solvent was removed. The condensed solvent was then mixed with the same amount of cold water $\left(0^{\circ} \mathrm{C}\right.$ to $\left.2{ }^{\circ} \mathrm{C}\right)$ and stirred for $2 \mathrm{~h}$. The resultant suspension was centrifuged at 20,000 rpm (Avanti J25centrifuge, JA 25.50 rotor; Beckman Coulter, Palo Alto, CA, USA) to remove the supernatant. Afterward, the pellets were suspended in ultrapure water, refrigerated, and freeze-dried.

\subsection{Characterization byTransmission Electron Microscopy (TEM), Scanning Electron Microscopy (SEM), and Zetasizer of RSV-SLN}

For TEM, $5 \mathrm{~mL}$ of each sample was placed on carbon formvar-coated 400-mesh spacing grids, left to become adsorbed for $5 \mathrm{~min}$, negatively stained with $2 \%$ sodium phosphotungstate for $45 \mathrm{~s}$, and allowed to dry. The grid was visualized by using a JEM 1400 electron microscope (JEOL-1230, Tokyo, Japan) at $80 \mathrm{kV}$. For the SEM, the samples were coated with aurum for 6 min using an Ion Sputter (JFC-1100, JEOL Ltd, Tokyo, Japan), and the thickness of cladding material was thinner than $20 \mathrm{~nm}$. Finally, after vacuuming, the shape and surface morphology of the samples were observed under S-4800 (Hitachi, Tokyo, Japan) scanning electron microscopes at an accelerating voltage of $20 \mathrm{kV}$. The magnification of the SEM images was 10,000 $\times$. The particle size and the zeta potential were determined at $25^{\circ} \mathrm{C}$ by photon correlation spectroscopy (Zetasizer Nano ZS, Malvern Instruments, Malvern, UK). For each sample, the measurements were repeated thrice.

\subsection{X-Ray Powder Diffraction (XRD) Analysis}

X-ray diffraction (XRD) patterns of pure RSV, RSV-SLN, and SLN were performed in order to characterize their crystallographic structure. The patterns were carried out with ' $X$ ' pert $P R O$, PANalytical instrument (Westborough, MA, USA), using $\mathrm{Cu}$-Ka rays with a voltage of $40 \mathrm{kV}$ and a current of $30 \mathrm{~mA}$, over the $2 \theta$ ranges $5-60^{\circ}$, with a step width $0.05^{\circ}$ and a scan time of 2.0 s per step. 


\subsection{Fourier-Transform Infrared (FTIR) Spectra of RSV-SLNs}

FTIR spectra were obtained on a CARY 50 spectrophotometer. Potassium bromide disc technique was employed to obtain the FTIR spectra of RSV, SLN-RSV, and SLN by the standard KBr disk method (sample/KBr = 1/100). The samples were ground gently with anhydrous $\mathrm{KBr}$ and compressed to form pellets. The spectrum was recorded in the range of $500-4000 \mathrm{~cm}^{-1}$ using TEN-SOR27(Bruker Co., Ettlingen, Germany).

\subsection{Animals}

Eight-week-old male C57BL/6J mice were purchased from Shanghai Laboratory Animal Research Center (SLAC, Shanghai, China) and kept in a controlled environment (12 h/12 h light/dark cycle, 08:00-20:00, temperature: $23 \pm 2{ }^{\circ} \mathrm{C}$, humidity: $60 \% \pm 5 \%$ ). They were randomly divided into four groups 1 week after acclimatization: (1) sedentary control group (SC; $n=6)$; (2) endurance exercise (EE; $n=6)$; (3) endurance exercise combined resveratrol supplementation (EE + RSV; $n=6)$; and (4) endurance exercise combined with RSV-SLN supplementation (EE + RSV-SLN; $n=6$ ). During the whole experiment, all the mice had free access to purified water and food and were weighed weekly. RSV and RSV-SLN were administered orally in EE + RSV group and EE + RSV-SLN group, respectively. RSV was administered at a dose of $25 \mathrm{mg} / \mathrm{kg}$ [4]. The amount of RSV-SLN was adjusted to be equal to that of the RSV treatment group. The SC and EE groups were performed with physiological saline solution as vehicle. Each treatment was administered once a day for 6 days/week for 8 weeks and performed $1 \mathrm{~h}$ before exercise. All the animal experimental protocols in this study were approved by the Animal Care and Use Committee of Shanghai Model Biology Research Center (Approval number SRCMO-IACUC No. 20140002).

\subsection{Exercise Protocol}

The whole training process was conducted on a motor treadmill (Jiangsu Saiangsi Biologic Technology Co., Jiangsu, China). After acclimating for 1 week, the mice exercised at low-moderate intensity for 8 weeks (speed initially at $10 \mathrm{~m} / \mathrm{min}, 120 \mathrm{~min}$ per day), and the speed was gradually increased to $20 \mathrm{~m} / \mathrm{min}$ until exhaustion. The exhaustive distance of the mice in each group was recorded. The mice in the control group were exposed to noise and handling, which were similar to those in the EE group, the EE + RSV group, and the EE + RSV-SLN group, to regulate exercise-associated stress.

\subsection{Indirect Calorimetry}

Indirect calorimetry was administered by computer-controlled automatic systems (Oxymax/ CLAMS-SC, Comprehensive Lab Animal Monitoring System, Columbus Instruments). The mice were tested in separate chambers to provide free water and ad libitum access to food. The velocity of indoor air passing through the chamber was $0.5 \mathrm{~L} / \mathrm{min}$. The exhaust gas of the combustion chamber was sampled for $1 \mathrm{~min}$ at an interval of $12 \mathrm{~min}$. Oxygen consumption and carbon dioxide production were estimated using $\mathrm{O}_{2}$ and $\mathrm{CO}_{2}$ sensors. Respiratory exchange rate (RER) was measured in terms of the volume of oxygen consumption and carbon dioxide production $\left(\mathrm{VO}_{2}=\mathrm{ViO}_{2} \mathrm{i}-\mathrm{VoO}_{2} \mathrm{O} ; \mathrm{VCO}_{2}\right.$ $=\mathrm{VoCO}_{2} \mathrm{O}-\mathrm{ViCO}_{2} \mathrm{i} ; \mathrm{RER}=\mathrm{VCO}_{2} / \mathrm{VO}_{2}$ ) [17]. Measurements were collected immediately after the endurance exercise challenge for 2 days.

\subsection{Tissue Sampling}

At the end of each manipulation, all the mice were subjected to fasting overnight and anesthetized by intraperitoneally injecting $2 \%$ sodium pentobarbital ( $6.5 \mathrm{mg} / 100 \mathrm{~g}$ body weight). The gastrocnemius (GAS) muscle tissues were completely excised and weighed individually. A portion of the GAS muscle tissue was kept for TEM or homogenized immediately to determine mitochondrial respiratory chain enzymes. The remaining portions were stored at $-80{ }^{\circ} \mathrm{C}$ until further analysis. 


\subsection{Ultrastructural Changes of Skeletal Muscle Tissues}

The cross-sectional GAS muscle tissues were immobilized in a fixed buffer ( $2 \%$ glutaraldehyde, $0.1 \mathrm{M}$ sodium cacodylate, $0.5 \%$ polyformaldehyde, $3 \mathrm{mM} \mathrm{CaCl}_{2}$, and $0.1 \mathrm{M}$ sucrose) for $4 \mathrm{~h}$ at $4{ }^{\circ} \mathrm{C}$, rinsed in PBS three times for $15 \mathrm{~min}$ at each time, dehydrated in ethanol, then acetone, and inserted in LX-112 (Ladd, Burlington, VT, USA). The tissues were then cut into $60-80 \mathrm{~nm}$ sections. The slides were stained by double staining with uranium lead ( $2 \%$ uranyl acetate and lead citrate saturated aqueous solution) and examined at $80 \mathrm{kV}$ by using a Tecnai 10 TEM (TECNAI G2 F20 S-TWIN, FEI, Oregon, USA). Digital images were captured with cameras (Olympus Soft Imaging Solutions, GmbH, Munster, Germany).

\subsection{Mitochondrial Respiratory Chain Enzyme Assays}

Citrate synthase (CS) activity, cytochrome (Cyt) c content, and adenosine triphosphatase (ATPase) activity in skeletal muscle were measured using the corresponding microplate assay kits (Nanjing Jiancheng Bioengineering Institute, Jiangsu, China) in accordance with the manufacturers' instructions. Absorbance was recorded with a TECAN microplate reader (TECAN, Sunrise, Mannedorf, Switzerland).

\subsection{Mitochondrial DNA (mtDNA) Content}

Quantitative real-time RT-PCR was performed to determine the ratio of a mitochondrial gene to a nuclear gene and to examine the mtDNA content in each sample in accordance with previously described methods with some modifications $[18,19]$. Mitochondrial NADH dehydrogenase subunit 1 (ND1) served as the mitochondrial mark, and platelet endothelial cell adhesion molecule-1 (PECAM-1) functioned as the nuclear reference mark (primer sequences in Table 1). Total DNA was extracted using a QIAamp DNA Mini kit in accordance with the manufacturer's instructions. A melting curve was obtained to ensure specific amplification, and the standard curve method was used for relative quantification. The ratio of mitochondrial ND1 to PECAM-1 was then calculated.

Table 1. List of primers used in the study.

\begin{tabular}{lll}
\hline Gene & Forward Primer $\left(\mathbf{5}^{\prime} \mathbf{-} \mathbf{3}^{\prime} \mathbf{)}\right.$ & Reverse Primer $\left(\mathbf{5}^{\prime} \mathbf{-} \mathbf{3}^{\prime} \mathbf{)}\right.$ \\
\hline ND1 & CCTATCACCCTTGCCATCAT & GAGGCTGTTGCTTGTGTGAC \\
PECAM-1 & ATGGAAAGCCTGCCATCATG & TCCTTGTTGTTCAGCATCAC \\
COX II & TTCAACACACTCTATCACTGGC & AGAAGCGTTTGCGGTACTCAT \\
COX $I V$ & TCACTGCGCTCGTTCTGATT & TGGCCTTCATGTCCAGCATT \\
CPT-1M & GCACACCAGGCAGTAGCTTT & CAGGAGTTGATTCCAGACAGGTA \\
CD 36 & ATGGGCTGTGATCGGAACTG & TTTGCCACGTCATCTGGGTTT \\
PGC-1 $\alpha$ & TATGGAGTGACATAGAGTGTGCT & CCACTTCAATCCACCCAGAAAG \\
NRF1 & AGCACGGAGTGACCCAAAC & TGTACGTGGCTACATGGACCT \\
Tfam & ATTCCGAAGTGTTTTTCCAGCA & TCTGAAAGTTTTGCATCTGGGT \\
Bnip3 & TCCTGGGTAGAACTGCACTTC & GCTGGGCATCCAACAGTATTT \\
Beclin-1 & ATGGAGGGGTCTAAGGCGTC & TCCTCTCCTGAGTTAGCCTCT \\
NIX & ATGTCTCACTTAGTCGAGCCG & CTCATGCTGTGCATCCAGGA \\
$\beta$-actin & ATTGCTGACAGGATGCAGAA & GCTGATCCACATCTGCTGGAA \\
\hline
\end{tabular}

\subsection{RNA Extraction and Semiquantitative RT-PCR}

Total RNA was isolated from the skeletal muscle tissues of each mouse by using Trizol (Invitrogen, Carlsbad, CA, USA) and reverse transcribed with a Superscript II kit (Invitrogen) in accordance with the manufacturer's recommendation. Table 1 shows the forward $(\mathrm{F})$ and reverse $(\mathrm{R})$ primers of mouse genes. PCR was conducted under the following conditions: $10 \mathrm{~min}$ at $94{ }^{\circ} \mathrm{C}, 30-35$ cycles at $94{ }^{\circ} \mathrm{C}$ $(30 \mathrm{~s}), 55^{\circ} \mathrm{C}(30 \mathrm{~s}), 72{ }^{\circ} \mathrm{C}(1 \mathrm{~min})$, and $10 \mathrm{~min}$ of incubation at $72{ }^{\circ} \mathrm{C}$. The mRNA levels were normalized to that of $\beta$-actin mRNA and quantified using the $2^{-\Delta \Delta \mathrm{Ct}}$ method. 


\subsection{Data and Statistical Analyses}

Data were expressed as mean \pm SEM. Differences in means were analyzed through one-way ANOVA. Significant level was set at $p<0.05$ (two sided). Data were examined using SPSS 19.0 (Chicago, IL, USA).

\section{Results}

\subsection{Characterization of SLN and RSV-SLN}

TEM images showed that SLN and RSV-SLN were both spherical in shape with smooth surfaces (Figure 1A,B). The discrete spheres were solid particles and had no aggregations. SEM analysis showed the smooth surface and the spherical morphology of the prepared SLN (Figure 1C) and RSV-SLN (Figure 1D), and these findings were consistent with previous reports [20]. Figure 1E showed the equivalent mean hydrodynamic diameter of RSV-SLN was about $112.5 \pm 10.3 \mathrm{~nm}$ with a narrow particle size distribution. Meanwhile, the zeta potential of RSV-SLN was $-24.7 \mathrm{mV}$ with a narrow polydispersity index $(\mathrm{PDI}=0.36 \pm 0.02)$ (Figure $1 \mathrm{~F})$.

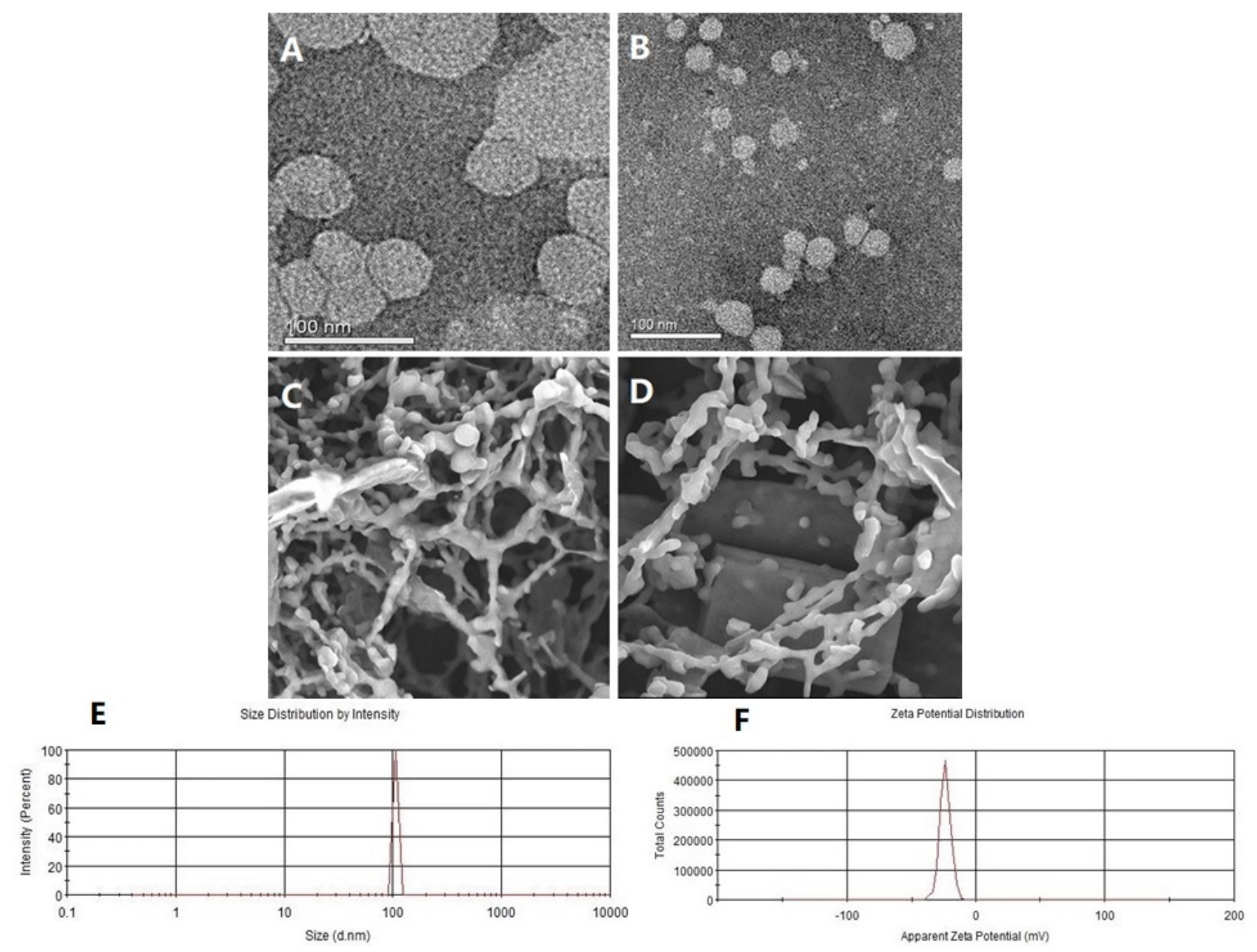

Figure 1. Characterization of RSV-SLN and SLN. TEM images of (A) SLN and (B) RSV-SLN after they were stained with one drop of $2 \%$ phosphotungstic acid. SEM images of (C) SLN and (D) RSV-SLN. (E,F) The size distribution and zeta potential for RSV-SLN. RSV: Resveratrol; SLN: solid lipid nanoparticles.

\subsection{X-Ray Diffraction Analysis of RSV-SLN}

XRD studies were performed in order to characterize drug status inside the SLN. As shown in Figure 2, the XRD pattern of the SLN showed the peaks at $2 \theta$ value of $21.55^{\circ}$ and $24.05^{\circ}$. The diffraction pattern of pure RSV showed different peaks at $2 \theta$ value of $16.36^{\circ}, 19.18^{\circ}, 22.67^{\circ}, 23.02^{\circ}$, and $27.67^{\circ}$, indicating highly crystalline nature structures. In addition, as for the XRD pattern of RSV-SLN, some 
similar characteristic peaks were also observed, which suggests that the RSV was present in crystalline state in SLN $[14,20]$.

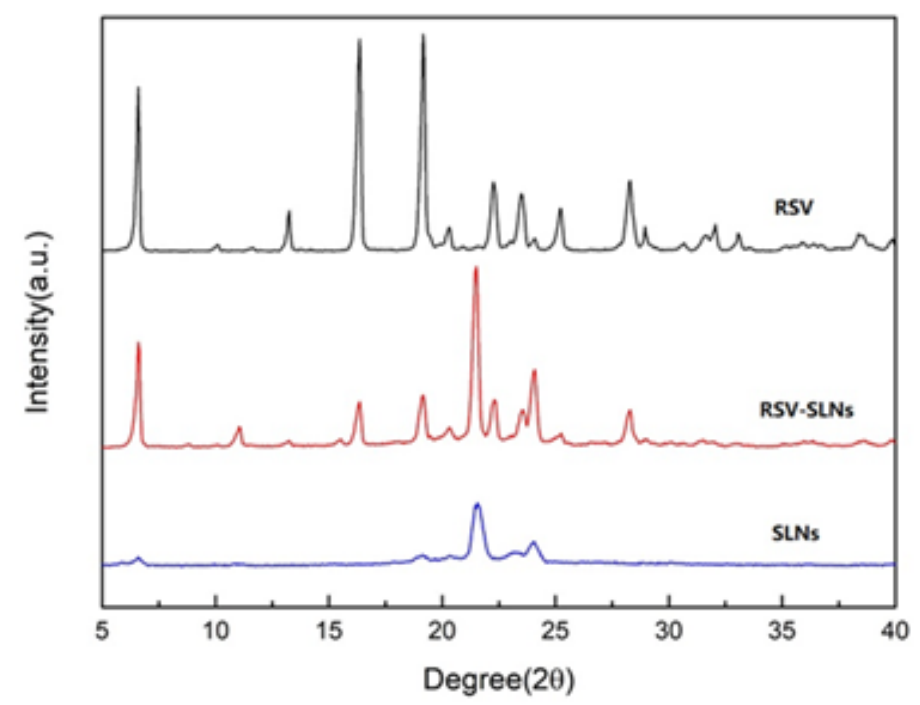

Figure 2. Powder X-ray diffraction patterns for RSV, RSV-SLN, and SLN.

\subsection{Fourier-Transform Infrared Raman (FTIR) Spectroscopy of RSV-SLN}

The FTIR spectra of RSV, RSV-SLN, and SLN (Figure 3) displayed no peak shifting and no loss of RSV peaks. In the FT-IR spectra, pure RSV has characteristic peaks around $1587 \mathrm{~cm}^{-1}$ representing benzene skeleton vibration. Peaks seen around $830 \mathrm{~cm}^{-1}$ represent the bending vibration of $\mathrm{C}=\mathrm{C}-\mathrm{H}$. The spectra of RSV-SLN showed a part of the functional characteristic peaks of RSV, probably because of RSV molecular dispersion or entrapment within SLN [16]. Moreover, no additional peaks were observed in the RSV-SLN spectra, indicating that the loading of RSV into SLN did not change the nature of SLN. Therefore, this study confirmed that no interaction occurred between RSV and the solid lipid component of SLN, indicating that RSV was compatible with SLN formulations; this finding was consistent with previous reports [21].

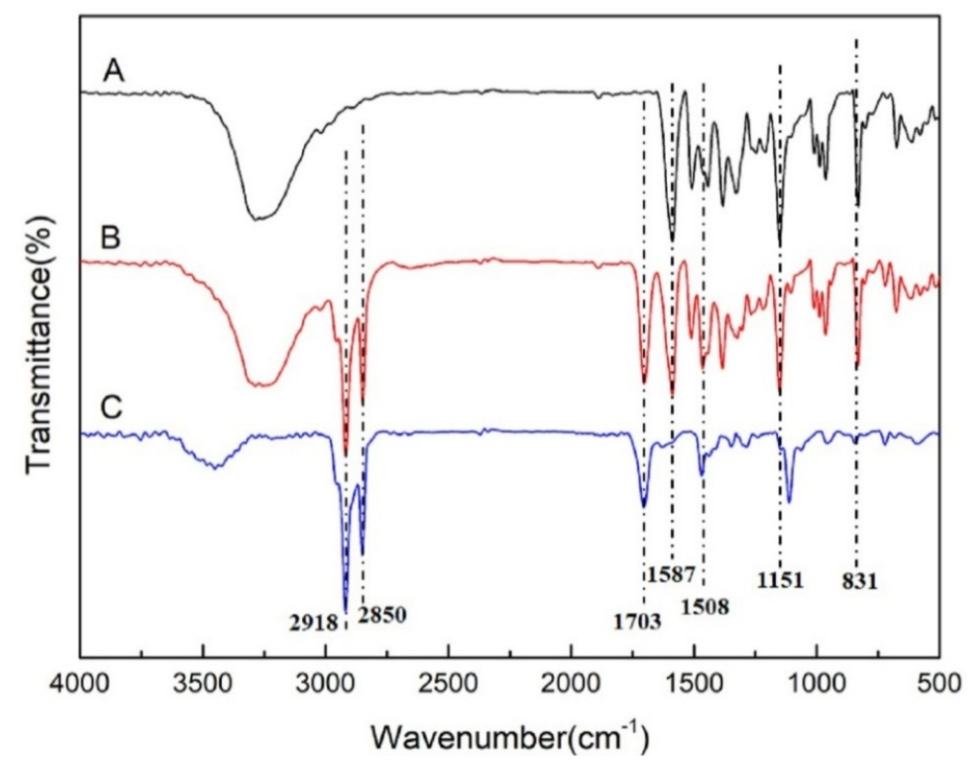

Figure 3. Fourier-transform infrared Raman (FTIR) spectra of RSV, RSV-SLN, and SLN; (A) FTIR spectra for RSV; (B) FTIR spectra for RSV-SLN; (C) FTIR spectra for SLN. 


\subsection{Effect of RSV-SLN Supplementation on Endurance Performance and Muscle Energy Utilization}

In this study, we observed the beneficial effects of RSV and RSV-SLN supplementation on excessive endurance exercise challenges and measured other biochemical indicators after 8 weeks of experimental intervention. The running distance to exhaustion significantly increased after RSV-SLN was administered (Figure 4A). The average exhaustive running distance of the EE + RSV-SLN group was $8617.78 \mathrm{~m}$, which was longer by $28.7 \%$ than that of the EE group. These results suggested that RSV-SLN supplementation could effectively enhance endurance performance.
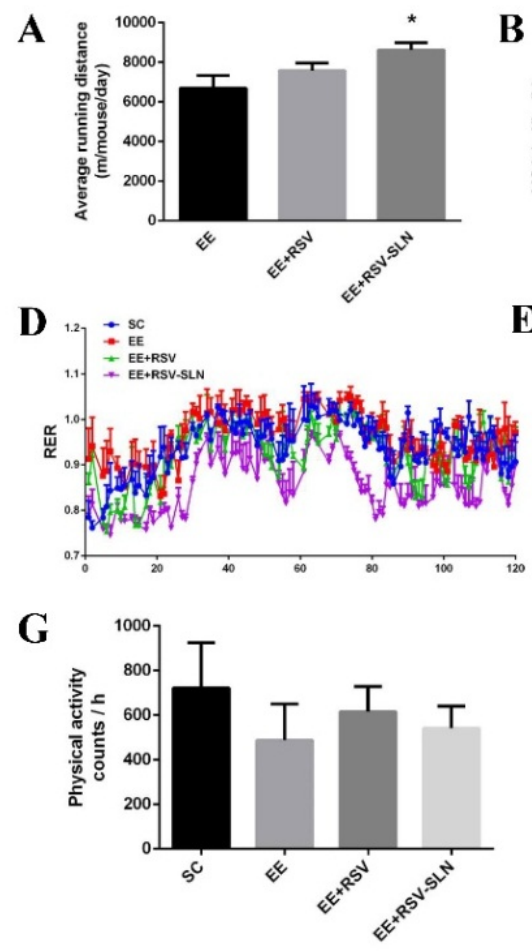

$\mathbf{E}$
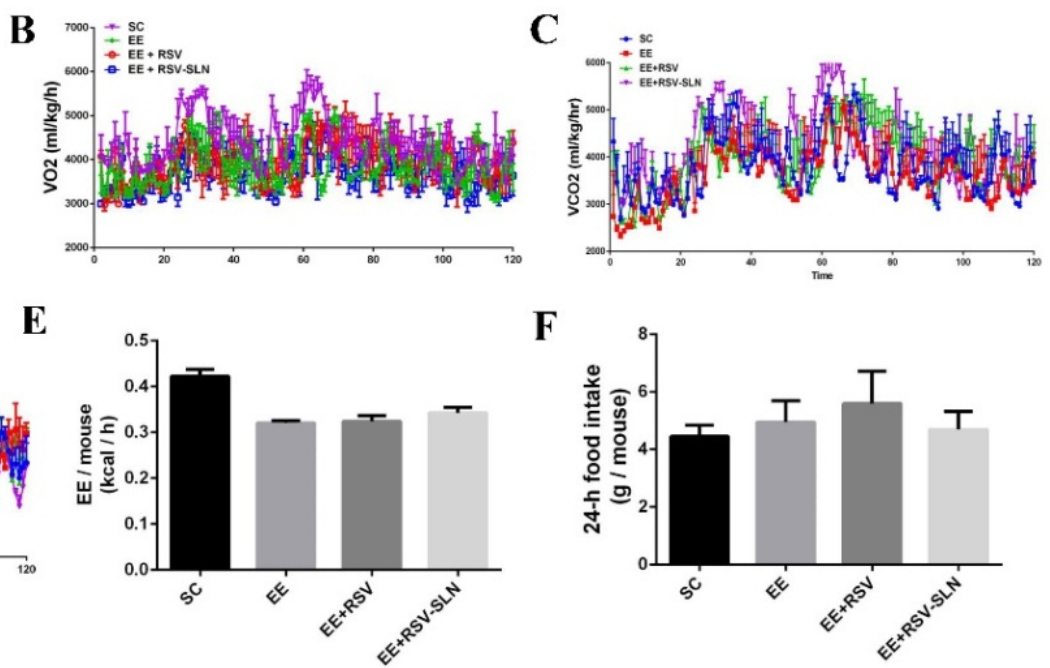

$\mathbf{F}$

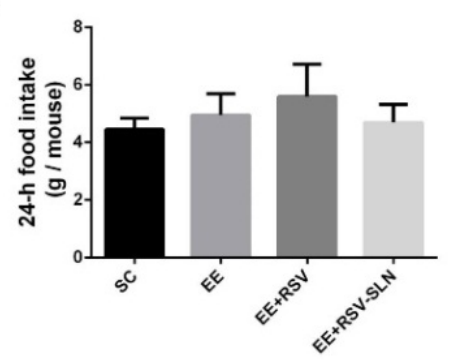

Figure 4. RSV-SLN supplementation enhances endurance performance. (A) Average running distances to the point of exhaustion. (B) Oxygen consumption $\left(\mathrm{VO}_{2}\right)$. (C) Carbon dioxide production $\left(\mathrm{VCO}_{2}\right)$. (D) Respiratory exchange ratio (RER). (E) Energy expenditure (EE). (F) Food intake for $24 \mathrm{~h}$. (G) Physical activity of mice from four groups. ${ }^{*} p<0.05 \mathrm{vs.} \mathrm{SC} \mathrm{(sedentary} \mathrm{control)} \mathrm{group.}$

Muscle energy utilization is essential for endurance exercise performance [22]. As such, we evaluated the muscle energy utilization of RSV-SLN-supplemented mice by measuring their oxygen utilization (Figure 4B), $\mathrm{CO}_{2}$ generation (Figure $4 \mathrm{C}$ ), and respiratory exchange ratio (Figure 4D). During low-moderate intensity exercise, the main energy source is fat. Consistent with this observation, our results showed that the RER decreased in the EE + RSV-SLN group. This result was accompanied by the enhancement of endurance exercise tolerance (Figure 4A). These data suggested that the amount of fat consumed by the mice with RSV-SLN supplementation was higher, and their dependence on lipid metabolism was greater than that of the EE controls because a lower RER typically reflects a substrate shift favoring fat metabolism. No significant difference was found in other indicators (Figure 4E-G).

\subsection{Effect of RSV-SLN Supplementation on the Muscular Ultrastructural Changes of Mice that Underwent Excessive Endurance Exercise}

Ultrastructural changes are shown in Figure 5A,B. The muscle fibers in the SC group showed normal characteristics under TEM images. The $\mathrm{M}$ line and the $\mathrm{Z}$ line were arranged orderly, and the mitochondrion ridge was clearly observed (Figure 5A). After excessive endurance exercise was completed, the $\mathrm{M}$ line and the $\mathrm{Z}$ line were arranged disorderly, and the mitochondria were damaged, 
swollen, and vacuolized (Figure 5B). However, the damaged ultrastructure was significantly improved after RSV and RSV-SLN treatments, especially RSV-SLN, were administered. The disordered M and Z lines were relieved, and the number of morphologically normal mitochondria increased (Figure 5C,D).
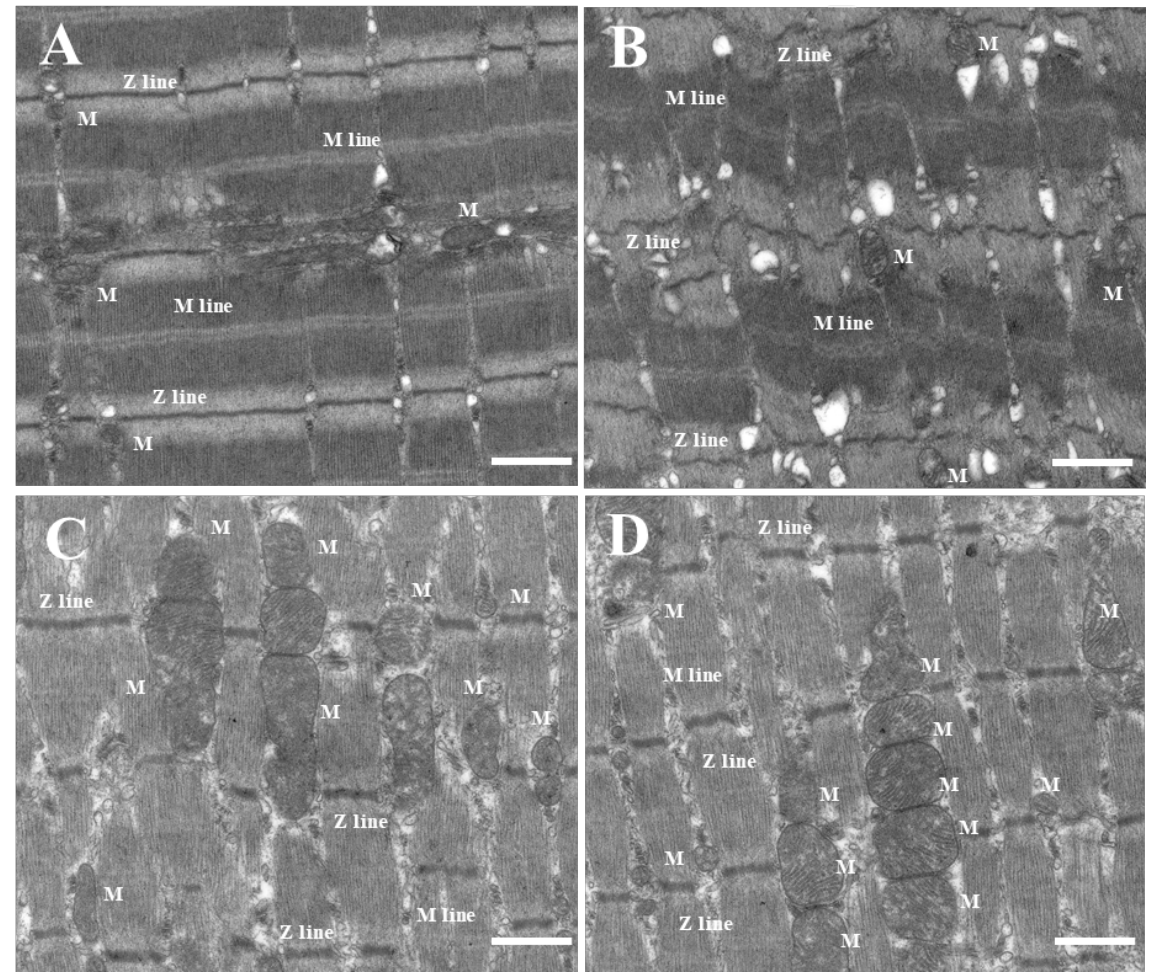

Figure 5. RSV-SLN alleviates the ultrastructural damage of skeletal muscles by excessive endurance exercise. Ultrastructure changes were shown via transmission electron microscopy from four groups of mice. Mt indicates mitochondria. The scale bars represent $500 \mathrm{~nm}$ in skeletal muscle tissues.

3.6. Effect of RSV-SLN Supplementation on Mitochondrial Function and Mitochondrial Long-Chain FA Translocase in Response to Excessive Endurance Exercise

We assume that, after RSV-SLN supplementation was administered, endurance performance was likely improved through the enhancement of mitochondrial function and mitochondrial long-chain FA translocase expression. As a result, energy insufficiency induced by long-term endurance exercise was alleviated. Enzymatic assays for mitochondrial respiratory chain complexes have been widely used to estimate mitochondrial function [23]. In our study, mitochondrial function was assessed by measuring the ATPase activity, Cyt c content, CS activity, and mRNA contents of mitochondrial complex IV (COX IV) and mitochondrial complex II (COX II) in the skeletal muscle tissue. Our results showed that long-term endurance exercise resulted in a decrease in the ATPase activity $(p<0.05)$, the CS activity $(p<0.05)$, and the transcription level of COX IV $(p<0.05)$ (Figure 6A,B,D), which induced an impairment in mitochondrial function. As expected, the CS activity and COX IV mRNA significantly increased after RSV-SLN was administered (Figure 6B,D), whereas the increase in the levels of ATPase activity, Cyt c content, and COX II mRNA was unnoticeable (Figure 6A,C,E). However, no changes in the mRNA levels of $C P T-1 M$ (a prominent isoform specific to skeletal muscle) and CD36 were observed in response to excessive exercise with or without RSV-SLN application (Figure 6F,G). 
A

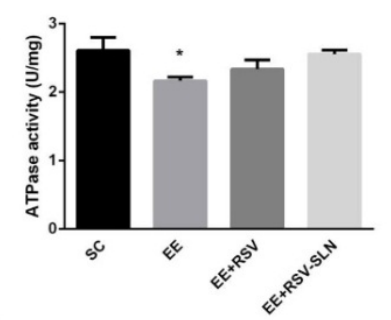

D

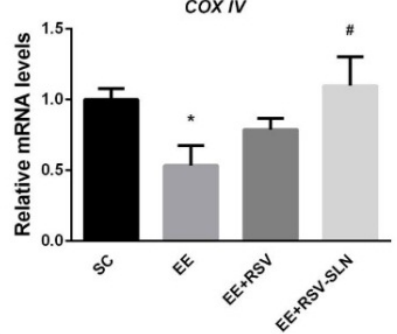

G

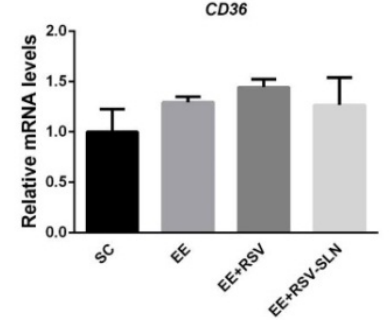

B

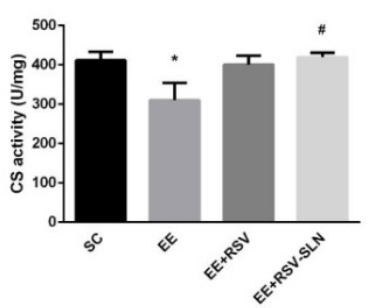

$\mathbf{E}$

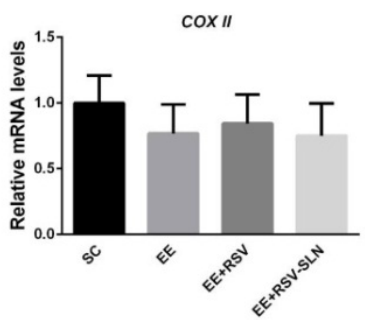

C

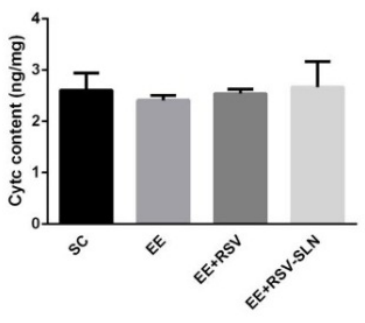

F

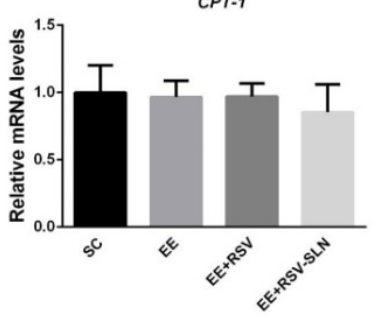

Figure 6. RSV-SLN alleviates mitochondrial dysfunction but does not alter mitochondrial long-chain FA translocase during excessive endurance exercise. (A) ATPase activity. (B) CS activity. (C) Cyt c content. (D,E) mRNA expression levels of COX IV and COX II. (F,G) mRNA expression levels of CPT-1 and CD36 in the skeletal muscle from four groups of mice. ${ }^{*} p<0.05 \mathrm{vs.} \mathrm{SC} \mathrm{group;}{ }^{\#} p<0.05 \mathrm{vs.} \mathrm{EE}$ group. FA: fatty acid; CS: citrate synthase; Cyt c: cytochrome c; COX IV: mitochondrial complex IV; COX II: mitochondrial complex II.

\subsection{Effect of RSV-SLN Supplementation on Mitochondrial QC in Response to Excessive Endurance Exercise}

The above results prompted us to explore the molecular mechanism involved in the amelioration of mitochondrial function through RSV-SLN supplementation in response to excessive endurance exercise. The healthy mitochondrial population is dynamic, and it shows a variable turnover rate in an active transcriptional process that links mitochondrial biogenesis to the degradation of damaged and senescent mitochondria via mitophagy [24]. Mitochondrial biogenesis and mitophagy were investigated to address mitochondrial function via the stimulation of the mitochondrial QC. Our results indicated that the mitochondrial DNA copy number decreased in mice that were subjected to excessive endurance exercise (Figure 7A), and this observation was consistent with the results on CS activity.

The activity of CS is a marker of mitochondrial content [25]. Furthermore, we measured the peroxisome proliferator receptor gamma coactivator-1 alpha (PGC-1 $\alpha)$, which is the main regulator of mitochondrial biogenesis, and its downstream transcription factors, namely, mitochondrial transcription factor A (Tfam) and nuclear respiratory factor 1 (NRF-1). Interestingly, the mRNA levels of PGC-1 $\alpha$, Tfam, and NRF-1 were significantly upregulated (Figure 7B-D) and incompatible with mtDNA content. As such, we aimed to explore the status of mitophagy. Consequently, we found that excessive endurance exercise significantly increased the expression levels of genes, including Bcl-2/adenovirus E1B 19 kD-interacting protein 3 (Bnip3), and Beclin-1, involved in mitophagy (Figure 7E,F). These results indicated that the increase in mitophagy, but not the suppression of mitochondrial biogenesis, resulted in the reduced mitochondrial content. 
A

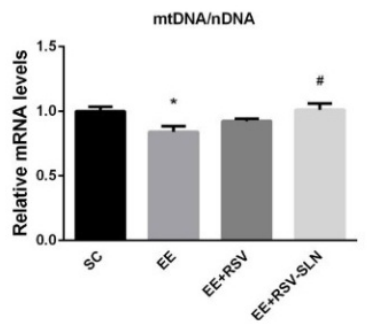

B

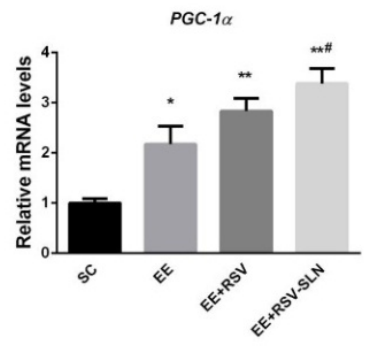

$\mathbf{E}$

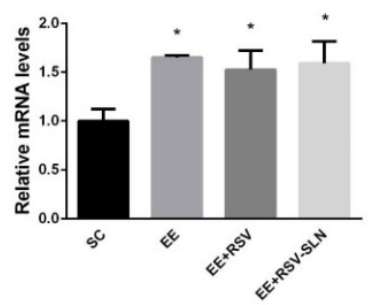

C

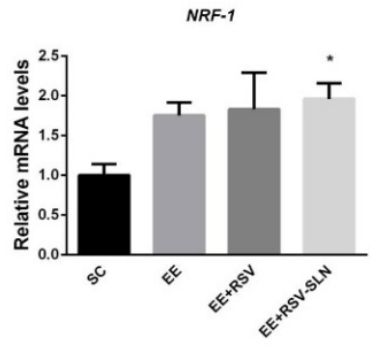

$\mathbf{F}$

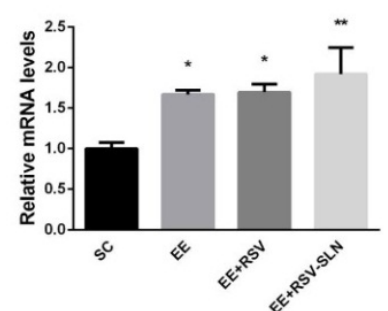

D

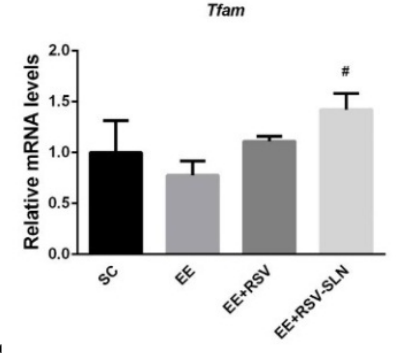

G

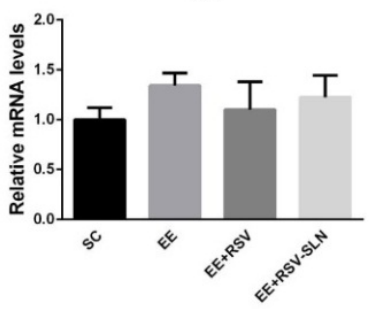

Figure 7. RSV-SLN elevates mitochondrial content by upregulating mitochondrial biogenesis and enhancing mitophagy capacity during excessive endurance exercise. (A) Mitochondrial DNA copy number (mtDNA). (B-D) The mRNA expression profile of mitochondrial biogenesis, PGC-1 $\alpha$, NRF1, and Tfam. (E-G) The mRNA expression profile of mitophagy, Bnip3, Beclin-1, and NIX in the skeletal muscle from four groups of mice. ${ }^{*} p<0.05$, and ${ }^{* *} p<0.01$ vs. SC group; ${ }^{*} p<0.05$ vs. EE group.

In accordance with the above observations, the mtDNA content of the mice exhausted through exercise following RSV-SLN administration increased (Figure 7A). Consistent with the increased abundance of mitochondria, the transcript levels of $P G C-1 \alpha$ and Tfam were significantly higher in the $\mathrm{EE}+\mathrm{RSV}-\mathrm{SLN}$ group than in the EE group. However, no changes were found in the transcription levels of Bnip3 and Beclin-1 or the mitophagic receptor NIP3-like protein X (NIX) in the skeletal muscle compared with those in the EE group, indicating that the increase in mitochondrial biogenesis contributed to the reversal of the mtDNA content but not to mitophagy. Altogether, our results implied a precise control between the production of functional mitochondria and the selective elimination of dysfunctional ones via RSV-SLN supplementation in mice that were subjected to excessive endurance exercise.

\section{Discussion}

In recent years, nanomaterials have been more used as drug delivery to improve their availability and efficiency. For instance, solid lipid nanoparticles have been introduced as an attractive alternative to traditional drug delivery systems. They are composed of physiological lipids and present many potential advantages, such as low toxicity, targeting ability, and bioavailability.

In this study, RSV-SLN was successfully prepared by emulsification and low-temperature solidification method. As observed via TEM and SEM, the RSV-SLN was spherical in shape with a narrow distribution. The mean particle size of RSV-SLNs with a nominal hydrodynamic diameter were about $112.5 \pm 10.3 \mathrm{~nm}$. The particle size with $100 \mathrm{~nm}$ range could improve cellular drug uptake and retention. The particle size measured by PCS was larger than those estimated by TEM. This is because PCS is used for hydrodynamic diameter examination, while TEM is used for characterization 
of particles in dried states. The zeta potential of RSV-SLN was $-24.7 \mathrm{mV}$, which could avoid the aggregation and keep the system a good physical dispersion. The XRD and FTIR spectra of the RSV-SLN revealed the drug status in the SLN, which confirmed that the RSV drug retained its chemical properties and successfully encapsulated in the SLN.

Exhaustive exercise produces physical stress, which transiently disrupts homeostasis [26], and the working skeletal muscles are the most directly affected organ during physical activity [27]. Excessive exercise can cause excessive accumulation of reactive oxygen species (ROS), which may lead to the imbalance between oxidative intermediates and antioxidant systems, and develop oxidative stress in muscles $[28,29]$. Some nutrients can limit mitochondrial ROS generation and relieve severe oxidative stress [3]. Effective ROS scavengers not only reduce mitochondrial damage but also interfere with the redox signaling involved in mitochondrial quality control. Therefore, scientists have been trying to investigate natural antioxidant extracts to relieve excessive oxidative damage induced by exercise and improve mitochondrial function.

RSV has been widely explored because of the discovery of antioxidant effects [30]. RSV protects mitochondrial function and mass against stress-induced oxidative damage [31]. However, its pharmacokinetic properties are unfavorable. Thus, incorporating RSV into nano formulation may be a useful and viable approach. As it is reported, RSV-SLN can display a sustained release effect, which will help to improve the practical delivery of RSV [16]. RSV molecules are highly stable when they are entrapped in SLN, thereby protecting RSV from degradation in biochemical reactions [32]. Additionally, the potential penetrating ability and cell uptake capacity of RSV-SLN affect the tissue accumulation of RSV that may be responsible for the effects of RSV-SLN in muscle tissue [15]. We mainly explored the improved protective effects and underlying mechanism of RSV-SLN on endurance performance and focused on the mitochondrial QC process. Our results showed that RSV-SLN applications significantly extended the running distance as a duration performance indicator. The three possibilities were as follows: first, RSV-SLN supplementation contributed to a substrate shift in favor of fat metabolism, which may be conducive to long-term energy that supplies working muscles. Second, RSV-SLN, as mitochondrial nutrition supplementation, can improve mitochondrial function through the indicators of ATPase and CS activity, thereby protecting against energy insufficiency during prolonged endurance exercise; this finding also agreed with our previous findings [33]. Finally, the application of RSV-SLN enhanced mitochondrial quality and quantity to match the increased energy requirements during long-term endurance exercise.

High mitochondrial content is closely related to endurance performance and muscular oxidative capacity. This study showed that RSV-SLN administration could reverse the decrease in the mtDNA content in response to excessive endurance exercise, and this decrease was parallel to CS activity and COX IV mRNA levels. Studies have indicated that the number of mitochondria is maintained through mitochondrial biogenesis and autophagy [34]. Once this cycle is disrupted, cells not only become susceptible to loss of energy regulation but also become subjected to oxidative damage secondary to dysfunctional mitochondria. However, the underlying role of RSV-SLN in improving the number of mitochondria during prolonged endurance exercise remains unclear.

A relatively small number of DNA-binding transcriptional regulators, such as PGC- $1 \alpha, N R F-1$, and Tfam, are essential for mitochondrial biogenesis [35]. Other studies have indicated that RSV administration can upregulate mitochondrial biogenesis by increasing PGC-1 $\alpha$ expression levels [33]. Similarly, despite the exhaustive training, RSV-SLN supplementation could still be positive and cause an increase in the mRNA expression levels of the upstream markers of mitochondrial biogenesis (PGC-1 $\alpha$ and Tfam) and downstream targets (CS and COX IV). Interestingly, in the EE group, the increase in the mRNA expression of mitochondrial proliferation regulators did not translate into an increase in mtDNA. The cause of these changes might be related to the aggravation of mitochondrial damage and mitophagy. Thus, mitophagy was often regarded as another center mechanism of mitochondrial quality and quantity control. 
Different mitophagy effectors, including the mitophagy receptors NIX, Bnip3, and PINK1/Parkin pathway, are involved in the selective clearance of mitochondria [36,37]. Bnip3 is an important mitochondrial redox sensor, which leads to cell death when cells are subjected to severe oxidative stress [38]. Under conditions such as hypoxia, Bnip3 and NIX may interact with Bcl-2 and release Beclin-1, resulting in the autophagic removal of mitochondria [36]. Our study showed that the transcriptional levels of Bnip3 and Beclin-1 were obviously upregulated in EE mice, suggesting that mitophagy that is induced by long-term endurance exercise might be dependent on the Bnip3/Beclin-1 pathway. We also observed a robust increase in the mRNA expression levels of Bnip3 and Beclin-1 after RSV or RSV-SLN supplementation when the mice were subjected to excessive exercise, indicating that RSV and RSV-SLN applications contributed to the clearing of damaged mitochondria. Taken together, our data demonstrated that the integration of mitochondrial biogenesis and mitophagy might be necessary to maintain energy supply during excessive endurance exercise; moreover, RSV-SLN could also partially relieve the exhaustive-exercise-induced mitochondrial response in vivo. Thus, we propose the probable mechanism as follows: first, drugs loaded in nanoparticle carriers can improve cellular drug uptake and retention via endocytosis in the cell membrane [39]. Second, the nanoparticle system may target mitochondrial sites via passive or active mechanisms that likely improve mitochondrial function [40]. Overall, we provided a novel perspective of RSV nanomaterial supplementation in improving endurance performance through a mitochondrion-mediated pathway (Figure 8).

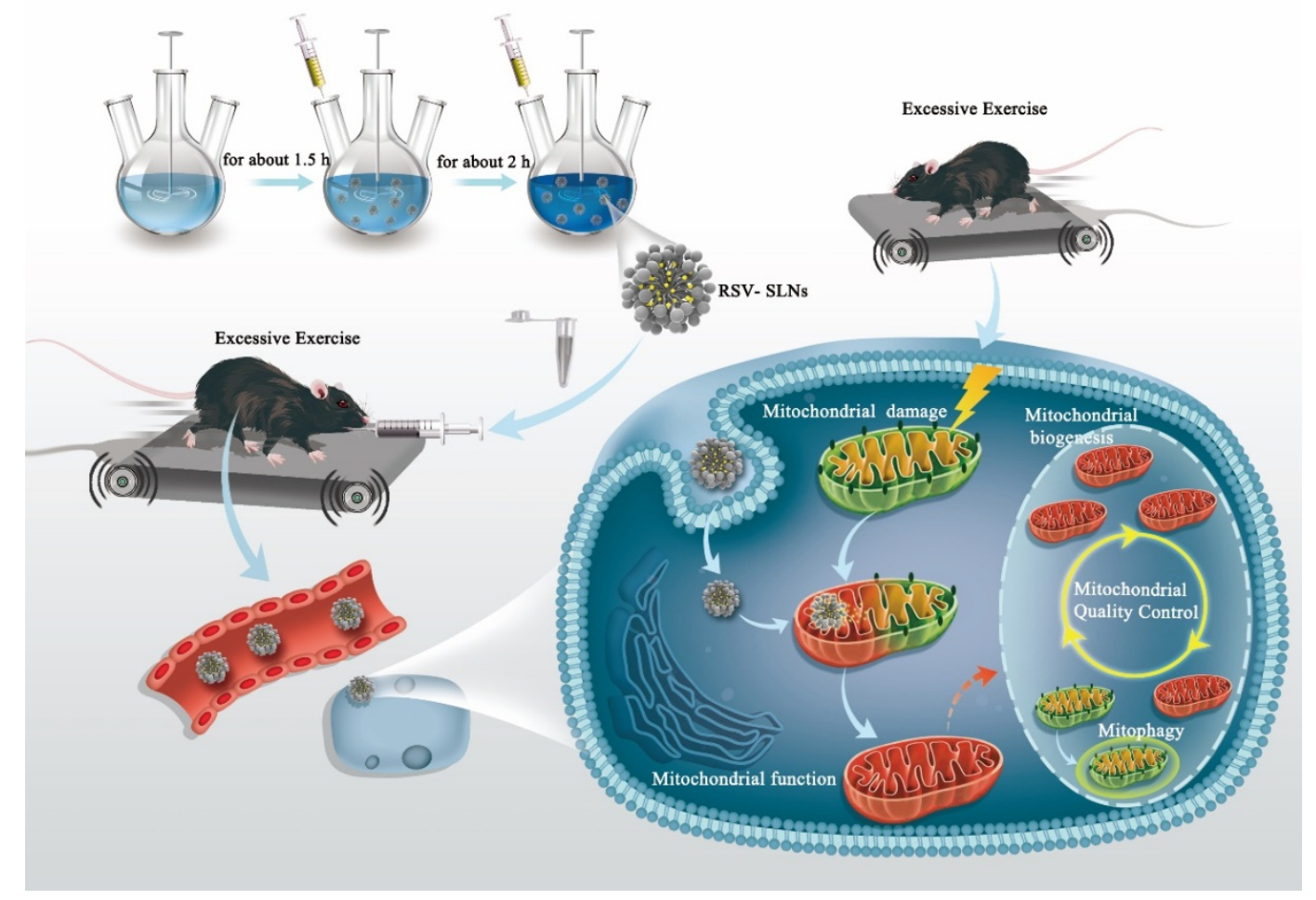

Figure 8. Schematic shows the mechanisms underlying RSV-SLNs to improve endurance performance by modulating mitochondrial quality control. RSV-SLNs were prepared through emulsification and low-temperature solidification. Results indicated that RSV-SLNs were superior to RSV because permeability and retention of the former were better than those of the latter. The possible biological mechanism is as follows: RSV-SLNs, as mitochondrial nutrients, can alleviate excessive endurance exercise-produced mitochondrial stress and improve mitochondrial function by ameliorating mitochondrial QC, thereby enhancing endurance performance and fatigue recovery. 


\section{Conclusions}

In summary, we revealed that an exhausting endurance exercise induces a decrease in mitochondrial quantity and quality through an imbalance between mitochondrial biogenesis and mitophagy, thereby impairing mitochondrial function. In turn, this impaired mitochondrial function leads to an insufficient energy supply. However, RSV-SLN administration might enhance mitochondrial function by ameliorating mitochondrial QC, thereby improving endurance performance, which was superior to RSV application. These results revealed an unexpected role for RSV-SLN compared with RSV in terms of linking nutrient deprivation to mitochondrial oxidant production through mitochondrial QC; moreover, a possible mechanism involved in improving endurance performance was identified. This study highlighted new possibilities for anti-physical-fatigue strategies.

Author Contributions: Conceptualization, J.S., T.L. and L.Q.; Data curation, Y.Z., Y.S. and Y.C.; Formal analysis, J.D.; Funding acquisition, J.S., Y.Z. and L.Q.; Methodology, S.L. and Q.H.; Project administration, L.Q.; Supervision, T.L.; Writing —original draft, J.S. and L.Q.; Writing—review and editing, T.L.

Funding: This research was funded by the National Natural Science Foundation of China (grant number 31600966, 31771313, and 31401019), the scientific fitness guidance project of National Sports General Administration of China (grant number 2017B026), Tengfei project of the Shanghai Municipal Sports Bureau (grant number 16T006) and the Fundamental Research Fund for the Central Universities of Tongji University.

Conflicts of Interest: The authors declare no conflict of interest.

\section{References}

1. Tanaka, M.; Baba, Y.; Kataoka, Y.; Kinbara, N.; Sagesaka, Y.M.; Kakuda, T.; Watanabe, Y. Effects of (-) -Epigallocatechin gallate in liver of an animal model of combined (physical and mental) fatigue. Nutrition 2008, 24, 599-603. [CrossRef] [PubMed]

2. Filler, K.; Lyon, D.; Bennett, J.; McCain, N.; Elswick, R.; Lukkahatai, N.; Saligan, L.N. Association of Mitochondrial Dysfunction and Fatigue: A Review of the Literature. BBA Clin. 2014, 1, 12-23. [CrossRef] [PubMed]

3. Huang, C.C.; Hsu, M.C.; Huang, W.C.; Yang, H.R.; Hou, C.C. Triterpenoid-Rich Extract from Antrodia camphorata Improves Physical Fatigue and Exercise Performance in Mice. Evid.-Based Complement. Alternat. Med. 2012, 2012, 1-8.

4. Sun, J.; Zhang, C.; Kim, M.; Su, Y.; Qin, L.; Dong, J.; Zhou, Y.; Ding, S. Early potential effects of resveratrol supplementation on skeletal muscle adaptation involved in exercise-induced weight loss in obese mice. BMB Rep. 2018, 51, 200-205. [CrossRef] [PubMed]

5. Busch, F.; Mobasheri, A.; Shayan, P.; Lueders, C.; Stahlmann, R.; Shakibaei, M. Resveratrol modulates interleukin-1beta-induced phosphatidylinositol 3-kinase and nuclear factor kappaB signaling pathways in human tenocytes. J. Biol. Chem. 2012, 287, 38050-38063. [CrossRef] [PubMed]

6. Zhao, H.; Niu, Q.; Li, X.; Liu, T.; Xu, Y.; Han, H.; Wang, W.; Fan, N.; Tian, Q.; Zhang, H.; et al. Long-term resveratrol consumption protects ovariectomized rats chronically treated with D-galactose from developing memory decline without effects on the uterus. Brain Res. 2012, 1467, 67-80. [CrossRef] [PubMed]

7. Walle, T.; Hsieh, F.; DeLegge, M.H.; Oatis, J.E.; Walle, U.K. High absorption but very low bioavailability of oral resveratrol in humans. Drug Metab. Dispos. 2004, 32, 1377-1382. [CrossRef]

8. Neves, A.R.; Lucio, M.; Lima, J.L.; Reis, S. Resveratrol in medicinal chemistry: A critical review of its pharmacokinetics, drug-delivery, and membrane interactions. Curr. Med. Chem. 2012, 19, 1663-1681. [CrossRef]

9. Doktorovova, S.; Silva, A.M.; Gaivão, I.; Souto, E.B.; Teixeira, J.P.; Martins-Lopes, P. Comet assay reveals no genotoxicity risk of cationic solid lipid nanoparticles. J. Appl. Toxicol. 2014, 34, 395-403. [CrossRef]

10. Doktorovova, S.; Souto, E.B.; Silva, A.M. Nanotoxicology applied to solid lipid nanoparticles and nanostructured lipid carriers-A systematic review of in vitro data. Eur. J. Pharm. Biopharm. 2014, 87, 1-18. [CrossRef]

11. Souto, E.B.; Doktorovová, S. Chapter 6-Solid lipid nanoparticle formulations pharmacokinetic and biopharmaceutical aspects in drug delivery. Methods Enzymol. 2009, 464, 105-129. [PubMed] 
12. Souto, E.B.; Müller, R.H. Lipid nanoparticles: Effect on bioavailability and pharmacokinetic changes. Handb. Exp. Pharmacol. 2010, 197, 115-141.

13. Teixeira, M.C.; Carbone, C.; Souto, E.B. Beyond liposomes: Recent advances on lipid based nanostructures for poorly soluble/poorly permeable drug delivery. Prog. Lipid Res. 2017, 68, 1-11. [CrossRef] [PubMed]

14. Jose, S.; Anju, S.S.; Cinu, T.A.; Aleykutty, N.A.; Thomas, S.; Souto, E.B. In vivo pharmacokinetics and biodistribution of resveratrol-loaded solid lipid nanoparticles for brain delivery. Int. J. Pharm. 2014, 474, 6-13. [CrossRef]

15. Mohseni, R.; ArabSadeghabadi, Z.; Ziamajidi, N.; Abbasalipourkabir, R.; RezaeiFarimani, A. Oral Administration of Resveratrol-Loaded Solid Lipid Nanoparticle Improves Insulin Resistance through Targeting Expression of SNARE Proteins in Adipose and Muscle Tissue in Rats with Type 2 Diabetes. Nanoscale Res. Lett. 2019, 14, 227. [CrossRef]

16. Gumireddy, A.; Christman, R.; Kumari, D.; Tiwari, A.; North, E.J.; Chauhan, H. Preparation, Characterization, and In vitro Evaluation of Curcumin- and Resveratrol-Loaded Solid Lipid Nanoparticles. AAPS PharmSciTech 2019, 20, 145. [CrossRef]

17. Kleiber, M. Metabolic turnover rate: A physiological meaning of the metabolic rate per unit body weight. J. Theor. Biol. 1975, 53, 199-204. [CrossRef]

18. Zhang, Q.; Zheng, J.; Qiu, J.; Wu, X.; Xu, Y.; Shen, W.; Sun, M. ALDH2 restores exhaustive exercise-induced mitochondrial dysfunction in skeletal muscle. Biochem. Biophys. Res. Commun. 2017, 485, 753-760. [CrossRef]

19. Pieters, N.; Koppen, G.; Smeets, K.; Napierska, D.; Plusquin, M.; De Prins, S.; Van De Weghe, H.; Nelen, V.; Cox, B.; Cuypers, A.; et al. Decreased mitochondrial DNA content in association with exposure to polycyclic aromatic hydrocarbons in house dust during wintertime: From a population enquiry to cell culture. PLoS ONE 2013, 8, e63208. [CrossRef]

20. Wang, W.; Zhang, L.; Chen, T.; Guo, W.; Bao, X.; Wang, D.; Ren, B.; Wang, H.; Li, Y.; Wang, Y.; et al. Anticancer Effects of Resveratrol-Loaded Solid Lipid Nanoparticles on Human Breast Cancer Cells. Molecules 2017, 22, 1814. [CrossRef]

21. Ramalingam, P.; Ko, Y.T. Improved oral delivery of resveratrol from N-trimethyl chitosan-g-palmitic acid surface-modified solid lipid nanoparticles. Colloids Surf. B Biointerfaces 2016, 139, 52-61. [CrossRef] [PubMed]

22. Fu, T.; Xu, Z.; Liu, L.; Guo, Q.; Wu, H.; Liang, X.; Zhou, D.; Xiao, L.; Liu, L.; Liu, Y.; et al. Mitophagy Directs Muscle-Adipose Crosstalk to Alleviate Dietary Obesity. Cell Rep. 2018, 23, 1357-1372. [CrossRef] [PubMed]

23. Lobo-Jarne, T.; Ugalde, C. Respiratory chain supercomplexes: Structures, function and biogenesis. Semin. Cell Dev. Biol. 2018, 76, 179-190. [CrossRef] [PubMed]

24. Palikaras, K.; Tavernarakis, N. Mitochondrial homeostasis: The interplay between mitophagy and mitochondrial biogenesis. Exp. Gerontol. 2014, 56, 182-188. [CrossRef] [PubMed]

25. Larsen, S.; Nielsen, J.; Hansen, C.N.; Nielsen, L.B.; Wibrand, F.; Stride, N.; Schroder, H.D.; Boushel, R.; Helge, J.W.; Dela, F.; et al. Biomarkers of mitochondrial content in skeletal muscle of healthy young human subjects. J. Physiol. 2012, 590, 3349-3360. [CrossRef] [PubMed]

26. Mastorakos, G.; Pavlatou, M. Exercise as a stress model and the interplay between the hypothalamus-pituitary-adrenal and the hypothalamus-pituitary-thyroid axes. Horm. Metab. Res. 2005, 37, 577-584. [CrossRef] [PubMed]

27. Hoene, M.; Franken, H.; Fritsche, L.; Lehmann, R.; Pohl, A.K.; Haring, H.U.; Zell, A.; Schleicher, E.D.; Weigert, C. Activation of the mitogen-activated protein kinase (MAPK) signalling pathway in the liver of mice is related to plasma glucose levels after acute exercise. Diabetologia 2010, 53, 1131-1141. [CrossRef]

28. Fogarty, M.C.; Hughes, C.M.; Burke, G.; Brown, J.C.; Trinick, T.R.; Duly, E.; Bailey, D.M.; Davison, G.W. Exercise-induced lipid peroxidation: Implications for deoxyribonucleic acid damage and systemic free radical generation. Environ. Mol. Mutagen. 2011, 52, 35-42. [CrossRef]

29. Alessio, H.M.; Hagerman, A.E.; Fulkerson, B.K.; Ambrose, J.; Rice, R.E.; Wiley, R.L. Generation of reactive oxygen species after exhaustive aerobic and isometric exercise. Med. Sci. Sports Exerc. 2000, 32, 1576-1581. [CrossRef]

30. Haohao, Z.; Guijun, Q.; Juan, Z.; Wen, K.; Lulu, C. Resveratrol improves high-fat diet induced insulin resistance by rebalancing subsarcolemmal mitochondrial oxidation and antioxidantion. J. Physiol. Biochem. 2015, 71, 121-131. [CrossRef] 
31. Sheu, S.J.; Liu, N.C.; Ou, C.C.; Bee, Y.S.; Chen, S.C.; Lin, H.C.; Chan, J.Y. Resveratrol stimulates mitochondrial bioenergetics to protect retinal pigment epithelial cells from oxidative damage. Investig. Ophthalmol. Vis. Sci. 2013, 54, 6426-6438. [CrossRef] [PubMed]

32. Qin, L.; Wang, W.; You, S.; Dong, J.; Zhou, Y.; Wang, J. In vitro antioxidant activity and in vivo antifatigue effect of layered double hydroxide nanoparticles as delivery vehicles for folic acid. Int. J. Nanomed. 2014, 9, 5701-5710. [CrossRef] [PubMed]

33. Lagouge, M.; Argmann, C.; Gerhart-Hines, Z.; Meziane, H.; Lerin, C.; Daussin, F.; Messadeq, N.; Milne, J.; Lambert, P.; Elliott, P.; et al. Resveratrol improves mitochondrial function and protects against metabolic disease by activating SIRT1 and PGC-1alpha. Cell 2006, 127, 1109-1122. [CrossRef] [PubMed]

34. Gottlieb, R.A.; Stotland, A. MitoTimer: A novel protein for monitoring mitochondrial turnover in the heart. J. Mol. Med. 2015, 93, 271-278. [CrossRef] [PubMed]

35. Scarpulla, R.C. Transcriptional paradigms in mammalian mitochondrial biogenesis and function. Physiol. Rev. 2008, 88, 611-638. [CrossRef] [PubMed]

36. Parikh, S.M.; Yang, Y.; He, L.; Tang, C.; Zhan, M.; Dong, Z. Mitochondrial function and disturbances in the septic kidney. Semin. Nephrol. 2015, 35, 108-119. [CrossRef] [PubMed]

37. Wei, H.; Liu, L.; Chen, Q. Selective removal of mitochondria via mitophagy: Distinct pathways for different mitochondrial stresses. Biochim. Biophys. Acta 2015, 1853, 2784-2790. [CrossRef]

38. Kubli, D.A.; Quinsay, M.N.; Huang, C.; Lee, Y.; Gustafsson, A.B. Bnip3 functions as a mitochondrial sensor of oxidative stress during myocardial ischemia and reperfusion. Am. J. Physiol. Heart Circ. Physiol. 2008, 295, H2025-H2031. [CrossRef]

39. Wang, J.; Wang, H.; Zhu, R.; Liu, Q.; Fei, J.; Wang, S. Anti-inflammatory activity of curcumin-loaded solid lipid nanoparticles in IL-1beta transgenic mice subjected to the lipopolysaccharide-induced sepsis. Biomaterials 2015, 53, 475-483. [CrossRef]

40. Choi, J.Y.; Gupta, B.; Ramasamy, T.; Jeong, J.H.; Jin, S.G.; Choi, H.G.; Yong, C.S.; Kim, J.O. PEGylated polyaminoacid-capped mesoporous silica nanoparticles for mitochondria-targeted delivery of celastrol in solid tumors. Colloids Surf. B Biointerfaces 2018, 165, 56-66. [CrossRef] 